\title{
Generalized boundary conditions for general relativity for the asymptotically flat case in terms of Ashtekar's variables
}

\author{
T. Thiemann* \\ Institute for Theoretical Physics, RWTH Aachen, \\ D-52074 Aachen, Germany
}

Preprint PITHA 93-31, August 93

\begin{abstract}
There is a gap that has been left open since the formulation of general relativity in terms of Ashtekar's new variables namely the treatment of asymptotically flat field configurations that are general enough to be able to define the generators of the Lorentz subgroup of the asymptotical Poincaré group. While such a formulation already exists for the old geometrodynamical variables, up to now only the generators of the translation subgroup could be defined because the function spaces of the fields considered earlier are taken too special. The transcription of the framework from the ADM variables to Ashtekar's variables turns out not to be straightforward due to the freedom to choose the internal $\mathrm{SO}(3)$ frame at spatial infinity and due to the fact that the non-trivial reality conditions of the Ashtekar framework reenter the game when imposing suitable boundary conditions on the fields and the Lagrange multipliers.
\end{abstract}

\section{Introduction}

Since the advent of the new canonical variables introduced by Ashtekar (四) the majority of related contributions have dealt, within the canonical treatment of gravity, with the case of a compact topology of the initial data hypersurface without boundary because it is technically simpler and although the major problem of general relativity, to define what is and how to construct an observable, is even more severe than for the case of an asymptotically flat topology since one then does not even have access to the well-known surface observables at spatial infinity (spi, not to be confused with the universal structure at spatial infinity which is called Spi ([3]).

Recently, the Killing-reduced model of spherically symmetric gravity with or without a Maxwell-field was shown to be a system for which the programme of canonical quantization can be carried out completely (畞). Since this is an isolated sytem

*hiemann@phys.psu.edu 
which is of physical interest only in the asymptotically flat context, the author was forced to consider the most general (spherically symmetric) field configurations and thus was lead out of the limitations given by the fall-off behaviour of the fields as proposed in ([1]). He found a genuinely complex ADM-momentum as defined for Ashtekar's formulation of general relativity even after the reality conditions have been imposed and figured out that this can be traced back to the fact that the symmetry generator corresponding to the vector constraint as defined in [1] is not differentiable if one considers fall-off conditions appropriate for the case of spherical symmetry. The problem was cured for spherical symmetry and by the way the more general fall-off conditions, valid for the full theory, were found, too.

This paper reports about these results :

In section 2 we review the framework given in [2] since there we find an explicit and consistent definition of the generators of the asymptotic Poincaré group. The calculations and definitions that follow will all be based on and motivated by the results of that paper.

In section 3 we propose a transcription of the definition of the function spaces of the basic fields from the geometrodynamical to Ashtekar's variables. The challenge is that there is now more asymptotic structure available since we are a priori free to choose the internal $\mathrm{SO}(3)$ frame. It turns out, however, that in order to recover the ADM-energy as given in [2] requires to fix the asymptotic internal frame. This is satisfactory because an 'SO(3)-charge' should not play any role in general relativity. The correction for the generating functional for the symplectomorphism from the old to the new variables compared to that in [1] is obtained.

In section 4 we derive the generators of the Poincaré group for the new variables. These are constructed from the constraint functionals by the requirement that they should be finite and functionally differentiable (together with the symplectic form) on the full phase space. Now, the virtue of the new variables is that the constraint functions turn out to be polynomial. Furthermore, with the function spaces considered in [1], the translation generators turn out to be also polynomial in the new variables. The main result is that if one wants to include boosts and rotations into the framework, the Poincaré generators necessarily become nonpolynomial. The constraint functions themselves, of course, remain polynomial so that the main advantage of the new variables is not invalidated.

However, this nonpolynomial nature of the Poincaré generators has an important consequence : it is not possible anymore, as stated frequently ([1]), to consider degenrate metrics (actually there is already a problem with degenerate metrics when imposing the reality conditions for Ashtekar's variables which involve the nonpolynomial spin-connection).

Altogether, what we do is just to do the symplectomorphism from the ADM phase space to the Ashtekar phase space correctly which in particular implies that on the constraint surface defined by the Gauss constraint all the results of [2] remain valid.

Section 5 checks that the Poincaré generators defined satisfy the correct gauge algebra, that they are Dirac observables ([5]) and that on the constraint surface one recovers the Lie algebra of the Poincare group modulo the well known supertranslation ambiguity.

Throughout it is assumed that the reader is familiar with the formulation of 
general relativity in terms of the new variables. For a review the reader is referred to [1]. We are using the abstract index notation.

\section{Geometrodynamics in the asymptotically flat context}

We give here mainly a compact account of the results obtained in [2] for later reference and since we want to compare the old and new formulation of general relativity. For more details the reader is referred to that paper :

The basic variables of the canonical formulation of general relativity for geometrodynamics are the intrinsic metric (or 1st fundamental form) $q_{a b}$ on the initial data hypersurface $\Sigma$ and its extrinsic curvature (or 2 nd fundamental form) $K_{a b}$ (more precisely the second basic variable is the momentum conjugate to $q_{a b}, p^{a b}:=(\operatorname{det}(q))^{1 / 2}\left(q^{a c} q^{b d}-\right.$ $\left.q^{a b} q^{c d}\right) K_{c d}$, where $q^{a b}$ is the inverse of $\left.q_{a b}\right)$. As usual for the discussion of asymptotic flatness ([6]), we will assume the topology of $\Sigma$ to be such that it is homeomorphic to the union of a compact set with a collection of 'ends' (i.e. asymptotic regions) where each end is homeomorphic to the complement of a compact ball in $R^{3}$. In the sequel we restrict ourselves to one end and mean by $\partial \Sigma \equiv S^{2}$ only spatial infinity of that end. The treatment of the remaining regions is identical.

Let us introduce a local frame in the neighbourhood of spi, denoted by $\left\{\bar{x}^{a}\right\}$, and we abbreviate the asymptotic 'spherical' variables by $r^{2}:=\bar{x}^{a} \bar{x}^{b} \delta_{a b}, \phi^{a}:=\bar{x}^{a} / r$ where $\phi^{a}$ coordinatizes spi (topologically $S^{2}$ ). We do not take this frame to be necessarily cartesian, but we assume that the transition matrix $\left(\partial\left(x_{\text {cart }}\right)^{a} / \partial \bar{x}^{b}\right.$ mediating to the cartesian one, $\left(x_{\text {cart }}\right)^{a}$, is of order $O(1)$.

Motivated by the appearence of the Schwarzschild solution in these coordinates one defines

$$
q_{a b} \rightarrow \bar{q}_{a b}+\frac{f_{a b}}{r}+O\left(1 / r^{2}\right)
$$

as $r \rightarrow \infty$ and it is assumed that the tensors $\bar{q}_{a b}, f_{a b}$ are smooth on spi $\left(\bar{q}_{a b}\right.$ is the euclidian metric in the asymptotic frame and it is assumed to be nondynamic, i.e. it is taken, as well as $\partial \Sigma$ as the '1st order part of the asymptotic structure at spi' $($ (《3) $)$.

In order to derive the asymptotic behaviour of $p^{a b}$ one seeks to make the symplectic structure

$$
\Omega:=\int_{\Sigma} d^{3} x d p^{a b}(x) \wedge d q_{a b}(x)
$$

well-defined (recall that for an integral curve $\gamma$ with parameter $\mathrm{t}$ on the phase space the gradient of $q_{a b}$ is defined by $d q_{a b}(x)\left[(\partial / \partial t)_{\mid \gamma}\right]:=\dot{q}_{a b}(x)_{\mid \gamma}$ and similar for the momentum variable). Furthermore, one wants to have the ADM-momentum to be non-vanishing (see formula (2.9)). This implies that the leading order part of $p^{a b}$ must be $O\left(1 / r^{2}\right)$. However, then the symplectic structure is logarithmically divergent in general. This can be cured by imposing that the leading order parts of the dynamical parts of the basic variables should have opposite parity. Since for an asymptotic translation lapse and shift are even functions at spi of order 1, the only way to have the ADM-4-momentum non-vanishing is to impose that $f_{a b}$ is even 
while $h^{a b}$ is odd (and smooth) at spi where

$$
p^{a b} \rightarrow \frac{h^{a b}}{r^{2}}+O\left(1 / r^{3}\right)
$$

(in order to see this, recall that the surface integrals are first evaluated at finite $\mathrm{r}$ and then one carries out the limit $r \rightarrow \infty$. We have then $d S_{a}:=\frac{1}{2} \epsilon_{a b c} d \bar{x}^{b} \wedge d \bar{x}^{c}=$ $n_{a} r^{2} d \mu$ where $\mu$ is the standard measure at spi and $n_{a}$ is the outward unit normal at spi, i.e. $d S_{a}$ is 'odd').

The constraint functionals of source-free general relativity in the old ADM-variables are given by

$$
\begin{aligned}
V_{a}\left[N^{a}\right] & =\int_{\Sigma} d^{3} x N^{a}\left(-2 D_{b} p_{a}^{b}\right) \\
C[N] & =\int_{\Sigma} d^{3} x N\left(\frac{1}{\sqrt{\operatorname{det}(q)}}\left(p^{a b} p_{a b}-\frac{1}{2}\left(p_{a}^{a}\right)^{2}\right)-\sqrt{\operatorname{det}(q)}\left({ }^{(3)} R\right)\right)
\end{aligned}
$$

called, respectively, the vector and the scalar constraint. Here we have introduced the components tangential $\left(N^{a}\right.$, the shift vector) and perpendicular ( $\mathrm{N}$, the lapse function) to the hypersurface $\Sigma$ of the foliation-defining vector field (with parameter t) and ${ }^{(3)} R$ is the scalar curvature of $\left(\Sigma, q_{a b}\right)$. It is understood that all indices are raised and lowered with the intrinsic metric whose unique torsion-free covariant differential is given by $\mathrm{D}$.

The vanishing of these constraint functionals defines the contraint surface $\bar{\Gamma}$ of the phase space. Since these constraints turn out to be first class in Dirac's terminology (more precisely, $\bar{\Gamma}$ is a coisotropic submanifold of $\Gamma$ ) they are to generate gauge transformations and so define the reduced phase space $\hat{\Gamma}$ by identifying points in $\bar{\Gamma}$ which lie on the same flow line of the Hamiltonian vector fields associated to the constraint functionals. It follows that one needs to compute Poisson-brackets with these constraint-functionals.

In order that one can compute Poisson-brackets of these constraint functionals with various functions on the phase space, they have to be

1) finite i.e. the integrals have to converge,

2) functionally differentiable.

Inserting the fall-off behaviour of our 2 basic fields into the equations (2.4), (2.5) one discovers that the integrals diverge, in general, if one not restricts the fall-off of the 'Lagrange-multipliers' $\left(N^{a}, N\right)$, too. There is no problem if one chooses them of, say, compact support but then the ADM-4 momentum (to be defined shortly) vanishes identically. In order to account for the generators of the asymptotic Poincaré group, the most general behaviour of lapse and shift that one would like to incorporate into the analysis is as follows:

$$
\begin{aligned}
N^{a} & \rightarrow a^{a}+\bar{\eta}_{b c}^{a} \varphi^{b} \bar{x}^{c}+\text { supertranslations } \\
N & \rightarrow a+\bar{q}_{a b} \beta^{a} \bar{x}^{b}+\text { supertranslations }
\end{aligned}
$$

Here $\left(a^{a}, a\right)$ are the parameters of the translation subgroup, $\varphi^{a}$ are rotation angles and $\beta^{a}$ are boost angles. These are vectors in the asymptotic $R^{3}$, i.e. constants, while the supertranslation parameters (to be specified in more detail shortly) are genuinely angle-dependent functions on $S^{2}$ (for more information about the supertranslation 
ambiguity, see ref. [3], [7]).

However, now the above generators of gauge transformations fail to be (manifestly) well-defined, nor are they functionally differentiable. This can be cured by using the following procedure :

Vary the basic fields and obtain expressions proportional to $\delta q_{a b}, \delta p^{a b}$. In doing this one picks up a surface integral. If the volume term of the variation is well-defined, it gives the searched for functional derivative of the functional we are seeking to make well defined. Subtract the surface term from the variation of the original constraint. If then finally the obtained surface term turns out to be exact, i.e. can be written as the variation of an ordinary surface integral, one has obtained this expression which is functionally differentiable and, if one is lucky, is already (manifestly) finite.

Let us exemplify this for the vector constraint : $D_{b} p_{a}^{b}$ is $O\left(1 / r^{3}\right)$ and even, so even for an asymptotic translation the integral in (2.4) diverges logarithmically. Upon variation we obtain

$$
\delta V_{a}\left[N^{a}\right]=\int_{\Sigma} d^{3} x\left(\mathcal{L}_{\vec{N}} q_{a b} \delta p^{a b}-\mathcal{L}_{\vec{N}} p^{a b} \delta q_{a b}\right)+2 \int_{\partial \Sigma} d S_{b} N^{a} \delta p_{a}^{b} .
$$

The volume part is obviously already finite : since $\vec{N}$ is an asymptotic Killing vector of $\bar{q}_{a b}$, we have $\mathcal{L}_{\vec{N}} q_{a b}=O\left(1 / r^{2}\right)$ odd and $O(1 / r)$ even for an asymptotic translation or rotation respectively while $\delta p^{a b}=O\left(1 / r^{2}\right)$ odd. Hence $\mathcal{L}_{\vec{N}} p^{a b}=O\left(1 / r^{3}\right)$ even and $O\left(1 / r^{2}\right)$ odd for an asymptotic translation or rotation respectively while $\delta q_{a b}=$ $O(1 / r)$ even. Hence, the integrand is either $O\left(1 / r^{4}\right)$ even or $O\left(1 / r^{3}\right)$ odd and so converges.

On the other hand $N^{a} \delta p_{a}^{b}=\delta\left(N^{a} p_{a}^{b}\right)$, so the surface term is indeed exact. Hence our candidate for a finite and functionally differentiable vector constraint arises from subtracting the corresponding counterterm :

$$
H_{a}\left[N^{a}\right]:=V_{a}\left[N^{a}\right]+2 \int_{\partial \Sigma} d S_{b} N^{a} p_{a}^{b}=\int_{\Sigma} d^{3} x \mathcal{L}_{\vec{N}} q_{a b} p^{a b}
$$

where we have carried out an integration by parts in the second step. This functional is differentiable by construction and luckily already finite since (by the same argument as above) the integrand is either $O\left(1 / r^{4}\right)$ even or $O\left(1 / r^{3}\right)$ odd. The surface term in (2.8) defines the ADM-momentum for an asymptotically constant shift, the integrand is $O(1)$ even and hence does not vanish :

$$
2 \int_{\partial \Sigma} d S_{b} N^{a} p_{a}^{b}=: a^{a} P_{a}
$$

wile for an asymptotic rotation we obtain

$$
2 \int_{\partial \Sigma} d S_{b} N^{a} p_{a}^{b}=: \varphi^{a} \bar{\eta}_{a b c} \bar{x}^{b} P^{c}
$$

which qualifies $J^{a}:=\bar{\eta}_{a b c} \bar{x}^{b} P^{c}$ as the asymptotic angular momentum.

For the scalar constraint one proceeds similarily. The difference is that due to the appearence of second spatial derivatives in ${ }^{(3)} R$ one has to do an integration by parts twice in order to arrive at a well-defined variation of the scalar constraint. The final result is given by $([2])$

$$
H[N]:=C[N]+2 \int_{\partial \Sigma} d S_{d} \sqrt{\operatorname{det}(q)} q^{a c} q^{b d}\left[N \bar{D}_{[c} q_{b] a}-\left(q_{a[b}-\bar{q}_{a[b}\right) \bar{D}_{c]} N\right]
$$


where $\bar{D}$ is the unique torsion-free covariant differential compatible with $\bar{q}_{a b}$. The first part of the surface integral is called the ADM-energy for an asymptotic timetranslation

$$
E:=2 \int_{\partial \Sigma} d S_{d} \sqrt{\operatorname{det}(q)} q^{a c} q^{b d} \bar{D}_{[c} q_{b] a}
$$

while the second part then vanishes. Otherwise we obtain the boost-generator

$$
\beta_{e} K^{e}:=\beta^{e}\left(E \bar{x}^{e}-2 \int_{\partial \Sigma} d S_{d} \sqrt{\operatorname{det}(q)} q^{a c} q^{b d}\left(q_{a[b}-\bar{q}_{a[b}\right) \delta_{c]}^{e}\right) .
$$

Note that since gauge transformations are those for which the generators $H, H_{a}$ induce identity transformations at spi on the constraint surface, they have to vanish then. Thus, only the odd part of the supertranslation parameters are non-vanishing in this case (the integrand without the Lagrange multipliers is even). In the seqel we will therefore consider the following fall-off behaviour of the Lagrange-multipliers :

$$
\begin{array}{r}
N^{a}:=T^{a}+R^{a}+S^{a}=T^{a}+\bar{\eta}^{a}{ }_{b c} \varphi^{b} \bar{x}^{c}+S^{a}, \\
N:=T+B+S=T+\beta_{a} \bar{x}^{a}+S
\end{array}
$$

where $\left(T, T^{a}\right)$ are $\mathrm{O}(1)$ even, i.e. generate an asymptotic translation in 4-dimensional space, $B, R^{a}$ are $O(r)$ odd, i.e. generate asymptotic boosts and rotations and finally $\left(S, S^{a}\right)$ are $O(1)$ and odd at least in leading order and thus generate the odd supertranslations. Moreover, the vector field $(T+B) \bar{n}^{a}+\left(T^{a}+R^{a}\right)$ is required to be a (10parameter) Killing vector field of the asymptotic spacetime metric $\bar{g}_{a b}=\bar{q}_{a b}-\bar{n}_{a} \bar{n}_{b}$ which implies, in particular, that $\mathcal{L}_{\vec{T}} q_{a b}$ is $O\left(1 / r^{2}\right)$ odd and that $\mathcal{L}_{\vec{R}} q_{a b}$ and $\mathcal{L}_{\vec{S}} q_{a b}$ are $O(1 / r)$ even. These properties will be used in the following.

\section{Connection dynamics in the asymptotically flat context}

We begin by transcribing the boundary conditions imposed on the ADM-variables to the new variables. In the following, latin letters from the beginning of the alphabet will denote tensor indices while latin letters from the middle of the alphabet will denote (internal) $\mathrm{SO}(3)$ indices.

Recalling that the triad 1 -form is the square-root of the 3 -metric, we expect the following fall-off behaviour

$$
e_{a}^{i} \rightarrow \bar{e}_{a}^{i}+\frac{f_{a}^{i}\left(\phi^{b}\right)}{r}+O\left(1 / r^{2}\right)
$$

where we have called the triad of the asymptotic 3-metric at spi $\bar{e}_{a}^{i}$. In order to investigate what the relation between the smooth tensors $f_{a b}$ and $f_{a}^{i}$ on spi is, we compute the 3 -metric

$$
q_{a b}=\delta_{i j} e_{a}^{i} e_{b}^{j}=\bar{q}_{a b}+2 \frac{\delta_{i j} \bar{e}_{(a}^{i} f_{b)}^{j}}{r}+O\left(1 / r^{2}\right)
$$

from which we infer

$$
f_{a b}=2 \delta_{i j} \bar{e}_{(a}^{i} f_{b)}^{j}
$$


This has the solution

$$
f_{a}^{i}=\left(\frac{1}{2} f_{a b}+\bar{\eta}_{a b c} v^{c}\right) \bar{e}_{i}^{b}
$$

where $v^{c}$ is a smooth vector field at spi.

Since the parity of $f_{a b}$ is even, $\bar{e}_{a}^{i}$ and $f_{a}^{i}$ must have equal parity. Note that we do not require $\bar{e}_{a}^{i}$ to be a constant (even if the asymptotic frame is cartesian). Also, the vector field $v^{a}$ is not constrained at all up to now.

Since (if the Gauss-constraint is satisfied) $K_{a}^{i}=K_{a b} e_{i}^{b}$ we need the asymptotic formula for the inverse triad. From the requirement $q^{a c} q_{c b}=\bar{q}_{b}^{a}$ we obtain unambiguosly

$$
q^{a b}=\bar{q}^{a b}-\bar{q}^{a c} \bar{q}^{b d} \frac{f_{c d}}{r}+O\left(1 / r^{2}\right)
$$

whence

$$
e_{i}^{a}=q^{a b} e_{b}^{i}=\bar{e}_{i}^{a}-\frac{\frac{1}{2} \bar{q}^{a c} f_{b c}-\bar{\eta}^{a}{ }_{b c} v^{c}}{r} \bar{e}_{i}^{b}
$$

Accordingly we obtain

$$
K_{a}^{i}=\frac{h_{a b}\left(\phi^{c}\right) \bar{e}_{i}^{b}}{r^{2}}+O\left(1 / r^{3}\right)
$$

and the smooth tensor $h_{a b}$ at spi has odd parity.

Finally, in order to derive the asymptotic formula for the Ashtekar-connection we need to determine the asymptotics for the spin-connection $\omega_{a}{ }_{j}^{i}$. First of all we extend the covariant derivative D to generalized tensors in the usual way, e.g.

$$
D_{a} e_{b}^{i}:=\bar{D}_{a} e_{b}^{i}-\Gamma_{a b}^{c} e_{c}^{i}+\omega_{a}{ }_{j}^{i} e_{b}^{j}=0
$$

where $\bar{D}$ is the covariant differential, extended to generalized tensors, that annihilates $\bar{e}_{a}^{i}$ and $\bar{q}_{a b}$. Note that this implies that the spin- and metric-connections of $e_{a}^{i}$ are $O\left(1 / r^{2}\right)$ while those of $\bar{e}_{a}^{i}$ are only $O(1 / r)$.

From the torsion-freeness requirement $\bar{D} \wedge e^{i}+\omega_{j}^{i} \wedge e^{j}=0$ we easily infer $(\bar{D}$ acts on tensor - and $\mathrm{SO}(3)$ indices !)

$$
\frac{r^{2} \bar{D}_{[a} \frac{f_{b]}^{i}}{r}+\lambda_{[a}^{i} j \bar{e}_{b]}^{j}}{r^{2}}+O\left(1 / r^{3}\right)=0
$$

where we have denoted the leading order part of the spin-connection by $\lambda_{a}{ }_{j}$. Since $\bar{D}_{b} f_{a}^{i}$ and $\bar{e}_{a}^{i}$ have opposite parity, this formula can only hold if $\lambda_{a}{ }_{j}^{i}$ has odd parity. Note :

This may also be checked explicitely : inverting formula (3.8), the analytic expression for the spin-connection in terms of the triads is given by (we define, as usual, $\Gamma_{a}^{i}:=$ $\left.1 / 2 \epsilon^{i j k} \omega_{a j k}\right)$

$$
\Gamma_{a}^{i}=-\frac{1}{2} \epsilon^{i j k} e_{j}^{b}\left(2 \bar{D}_{[a} e_{b]}^{k}+e_{k}^{c} e_{a}^{l} \bar{D}_{c} e_{b}^{l}\right)
$$

from which we immediately derive

$$
\lambda_{a}^{i}=-r^{2} \frac{1}{2} \epsilon^{i j k} \bar{e}_{j}^{b}\left(2 \bar{D}_{[a} \frac{f_{b]}^{k}}{r}+\bar{e}_{k}^{c} \bar{e}_{a}^{l} \bar{D}_{c} \frac{f_{b}^{l}}{r}\right) .
$$

Since this is an even polynomial in $\bar{e}_{a}^{i}$ and $f_{a}^{i}$ (both of which have equal parity), homogenous of 1st degree in the spatial derivatives, and $\mathrm{r}$ is an even function at spi, 
expression (3.11) is altogether an odd quantity. Hence, it follows directly from the formula for the Ashtekar-connection, $A_{a}^{i}=\Gamma_{a}^{i}+i K_{a}^{i}$, that its leading order part $G_{a}^{i}$ where

$$
A_{a}^{i}=\frac{G_{a}^{i}}{r^{2}}+O\left(1 / r^{3}\right)
$$

has definite parity if and only if the leading order part of $K_{a}^{i}$ is odd, i.e. if and only if $\bar{e}_{a}^{i}$ is even.

Since the 2 nd variable of the new canonical formulation of general relativity is given by $E_{i}^{a}:=\sqrt{\operatorname{det}(q)} e_{i}^{a}$, i.e.

$E_{i}^{a}=\bar{E}_{i}^{a}-\sqrt{\operatorname{det}(\bar{q})} \frac{1 / 2 \bar{q}^{a c} f_{b c}-\bar{\eta}_{b c}^{a} v^{c}+\bar{q}^{b c} f_{b c} \bar{q}_{b}^{a}}{r} \bar{e}_{i}^{b}+O\left(1 / r^{2}\right)=: \bar{E}_{i}^{a}+\frac{F_{a}^{i}}{r}+O\left(1 / r^{2}\right)$

we see that the next to leading order part $F_{a}^{i}$ has definite parity if and only if we set $v^{a}=0$. This parity is, fortunately, even if we want $G_{a}^{i}$ to have definite (odd) parity. Hence, it is possible to choose parity conditions in such a way that $A_{a}^{i} E_{i}^{a}$ is asymptotically an odd scalar density without violating that $E_{i}^{a}$ and $A_{a}^{i}$ 'have to come from $q_{a b}$ and $K_{a b}$ ', however, these parity conditions are rather awkward : they imply that $E_{i}^{a}$ becomes asymptotically a pseudo-vector density while $A_{a}^{i}$ is asymptotically a true covector. Moreover, parity conditions cannot be reversed if one wants to make the Ashtekar formulation meaningful in the asymptotically flat context, just as for the old ADM-formulation.

As one can check, the symplectic form is already finite thanks to our parity conditions (we set the gravitational coupling constant equal to one in the sequel) :

$$
\Omega:=\int_{\Sigma} d^{3} x(-i) d E_{i}^{a} \wedge d A_{a}^{i} \rightarrow \int_{\Sigma} d^{3} x\left[\frac{d F_{i}^{a} \wedge d G_{a}^{i}}{r^{3}}+O\left(1 / r^{4}\right)\right]
$$

Recalling that the Ashtekar-action arises from a complex canonical transformation, we ask if it is still true that its generating functional is given by $\int_{\Sigma} d^{3} x E_{i}^{a} \Gamma_{a}^{i}$ which was the case for the boundary conditions discussed by Ashtekar ([1]). But it is obvious that this functional diverges logarithmically without further specification. Indeed, using the explicit expression (3.10) we compute

$$
2 E_{i}^{a} \Gamma_{a}^{i}=\epsilon^{a b c} e_{c}^{i} \bar{D}_{b} e_{a}^{i} \rightarrow \frac{r^{2} \epsilon^{a b c} \bar{e}_{c}^{i} \bar{D}_{b} \frac{f_{a}^{i}}{r}}{r^{2}}+O\left(1 / r^{3}\right)
$$

and the first term vanishes since $\bar{D}_{a} \bar{e}_{b}^{i}=0$ and $f_{a b}$ is symmetric, while the second has no definite parity. We can cure this simply by applying the following trick : using that $\bar{D}_{a} \bar{e}_{b}^{i}=0$ we can write

$$
\begin{aligned}
& 2 E_{i}^{a} \Gamma_{a}^{i}=\epsilon^{a b c} e_{c}^{i} \bar{D}_{b} e_{a}^{i} \\
= & \epsilon^{a b c} e_{c}^{i} \bar{D}_{b}\left(e_{a}^{i}-\bar{e}_{a}^{i}\right) \\
= & \bar{D}_{b}\left(\epsilon^{a b c} e_{c}^{i}\left(e_{a}^{i}-\bar{e}_{a}^{i}\right)\right)-\epsilon^{a b c}\left(\bar{D}_{b} e_{c}^{i}\right)\left(e_{a}^{i}-\bar{e}_{a}^{i}\right) \\
= & \partial_{b}\left(\epsilon^{a b c} e_{c}^{i}\left(e_{a}^{i}-\bar{e}_{a}^{i}\right)\right)-\epsilon^{a b c}\left(\bar{D}_{b} e_{c}^{i}\right)\left(e_{a}^{i}-\bar{e}_{a}^{i}\right) .
\end{aligned}
$$

The 2 nd term in the last line is manifestly $O\left(1 / r^{3}\right)$ and odd, so gives a convergent integral, while the first yields a surface integral. Accordingly, we propose to define 
the generating functional for the spin connection by

$$
\int_{\Sigma} d^{3} x E_{i}^{a} \Gamma_{a}^{i}-\frac{1}{2} \int_{\partial \Sigma} d S_{b}\left(\epsilon^{a b c} e_{c}^{i}\left(e_{a}^{i}-\bar{e}_{a}^{i}\right)\right)
$$

and we can quickly check that this expression is indeed functionally differentiable and that the functional derivative is precisely the spin-connection. To see this, vary the integrand of the volume integral to obtain 2 terms

$$
\delta\left(E_{i}^{a} \Gamma_{a}^{i}\right)=\Gamma_{a}^{i} \delta E_{i}^{a}+E_{i}^{a} \delta \Gamma_{a}^{i}
$$

The first is already the required one while the second can be written (after tedious calculations) as

$$
E_{i}^{a} \delta \Gamma_{a}^{i}=\frac{1}{2} \partial_{a}\left(\epsilon^{a b c} e_{b}^{i} \delta e_{c}^{i}\right) .
$$

Varying the surface term on the other hand (recall that the asymptotic triad is non-dynamical, i.e. $\left.\delta \bar{e}_{a}^{i}=0\right)$ yields

$$
\begin{aligned}
& -\frac{1}{2} \int_{\partial \Sigma} d S_{b}\left(\epsilon^{a b c}\left[\left(e_{a}^{i}-\bar{e}_{a}^{i}\right) \delta e_{c}^{i}+e_{c}^{i} \delta e_{a}^{i}\right]\right. \\
= & -\frac{1}{2} \int_{\partial \Sigma} d S_{a} \epsilon^{a b c} e_{b}^{i} \delta e_{c}^{i}
\end{aligned}
$$

where the first term in the first line of (3.20) has dropped out because it is finite and odd. Comparing this result with eq. (3.19) we observe that the 2 terms exactly cancel.

\section{The Poincaré-generators for the new variables}

The final task is now to make the constraints convergent and functionally differentiable. We begin with the Gauss-constraint :

$$
\mathcal{G}_{i}:=\bar{D}_{a} E_{i}^{a}+\epsilon_{i j k} A_{a}^{j} E_{k}^{a} .
$$

The first term is $O\left(1 / r^{2}\right)$ while the second is $O\left(1 / r^{3}\right)$ and the whole expression is odd. Hence we need only worry about the first term whose variation, when integrated against the Lagrange-multiplier $\Lambda^{i}$, is just given by

$$
\int_{\partial \Sigma} d S_{a} \Lambda^{i} \delta E_{i}^{a}
$$

Recalling that $\Lambda^{i}$ is nothing else than the time component of the self-dual part of the 4-dimensional spin-connection, we require that it is $O\left(1 / r^{2}\right)$ for a symmetry transformation. Hence, the Gauss-constraint is already finite and functionally differentiable if we further require that $\Lambda^{i}$ be even. This parity condition is also consistent with the interpretation of $\Lambda^{i}=T^{a}{ }^{(4)} A_{a}$ because it is the contraction of an odd vector with an odd covector. There is thus no need for the $\mathrm{SO}(3)$-charge

$$
Q:=\int_{\Sigma} d S_{a} E_{i}^{a} \Lambda^{i}
$$


which then vanishes anyway due to parity and whose variation is also zero. Note, however, that for model systems which do not have access to parity (e.g. 베) the $\mathrm{SO}(3)$-charge is non-vanishing and gives a spurious observable which is fortunately unnecessary (the $\mathrm{SO}(3)$-charge should play no role in general relativity).

The treatment of the vector and scalar constraint turns out to be much more difficult and differs from the one given in [1]. This is to be expected because the generating functional (3.17) of the canonical transformation to the new canonical variables as given here is different from the one given in [1]. It gives rise to additional terms in the action, both in the volume and the surface part.

It turns out to be the easiest strategy in finding the necessary modifications to take the old ADM-action in its manifestly finite and functionally differentiable version and then to express it in terms of the new variables. We cannot expect it to remain well-defined because there are now 'more' variables. However, using the gauge constraint, we must be able to restrict the variations of the constraints to those of the ADM phase space. This is the outline of the idea, let us now come to the technical part of it.

Let us start with the expression (2.8), the generator of asymptotical spatial translations and rotations. We have

$$
\begin{aligned}
H_{a}\left[N^{a}\right] & =\int_{\Sigma} d^{3} x p^{a b} \mathcal{L}_{\vec{N}} q_{a b}=-\int_{\Sigma} d^{3} x p_{a b} \mathcal{L}_{\vec{N}} q^{a b} \\
& =-\int_{\Sigma} d^{3} x p_{a b} \mathcal{L}_{\vec{N}}\left(\frac{E_{i}^{a} E_{i}^{b}}{\operatorname{det}(q)}\right) \\
& =-\int_{\Sigma} d^{3} x \frac{p_{a b}}{\operatorname{det}(q)}\left[2 E_{i}^{b} \mathcal{L}_{\vec{N}} E_{i}^{a}-E_{i}^{a} E_{i}^{b}\left(E^{-1}\right){ }_{c}^{j} \mathcal{L}_{\vec{N}} E_{j}^{c}\right] \\
& =-2 \int_{\Sigma} d^{3} x\left[\left(K_{a b}-K q_{a b}\right) e_{i}^{b}+K e_{a}^{i}\right] \mathcal{L}_{\vec{N}} E_{i}^{a} \\
& =-2 \int_{\Sigma} d^{3} x K_{a}^{i} \mathcal{L}_{\vec{N}} E_{i}^{a}
\end{aligned}
$$

where we have used the elementary fact that $p^{a b} \mathcal{L}_{\vec{N}} q_{a b}=p^{a b} \mathcal{L}_{\vec{N}}\left(q_{a c} q_{b d} q^{c d}\right)=2 p^{a b} \mathcal{L}_{\vec{N}} q_{a b}+$ $p_{a b} \mathcal{L}_{\vec{N}} q^{a b}=-p_{a b} \mathcal{L}_{\vec{N}} q^{a b}$ that $\operatorname{det}(q)=\operatorname{det}\left(E_{i}^{a}\right)$ and the definition of the momentum conjugate to the 3-metric in terms of the extrinsic curvature (it seems as if up to now we have only used differential geometric identities but actually we made use of the Gauss-constraint in order to replace the variable $K_{a b}$ by $K_{a}^{i}$ ).

Finally we use the reality conditions in order to write (3.4) in terms of the Ashtekarconnection :

$$
H_{a}\left[N^{a}\right]=2 i \int_{\Sigma} d^{3} x\left(A_{a}^{i}-\Gamma_{a}^{i}\right) \mathcal{L}_{\vec{N}} E_{i}^{a}
$$

Noting that $E_{i}^{a}$ is a covector density of weight one, we have $\mathcal{L}_{\vec{N}} E_{i}^{a}=\bar{D}_{b} N^{b} E_{i}^{a}+$ $N^{b} \bar{D}_{b} E_{i}^{a}-E_{i}^{a} \bar{D}_{b} N^{a}$ and this expression is obviously $O(1 / r)$ odd for an asymptotic translation while it is $\mathrm{O}(1)$ even for an asymptotic rotation (note that this expression is a genuine generalization to arbitrary asymptotic frames of the formula for the cartesian frame since $\bar{D}$ acts also on internal indices; for the cartesian frame the metric- and spin connection vanish asymptotically so that we recover the usual formula in this case; also, for usual tensors there is no difference. The motivation for this generalization is that we want to keep $\partial_{a} E_{i}^{b}=O\left(1 / r^{2}\right)$ when passing to an arbitrary asymptotic frame). Hence the integral (4.5) diverges in either of these 
cases ! To see how this comes about, we compute the Lie-derivative of the twice densitized inverse asymptotic metric in terms of the electric fields

$$
0=\mathcal{L}_{\vec{N}}\left(\operatorname{det}(\bar{q}) \bar{q}^{a b}\right)=2 \bar{E}_{i}^{(a} \mathcal{L}_{\vec{N}} \bar{E}_{i}^{b)}
$$

which vanishes since $\vec{N}$ is an asymptotic Killing vector of $\bar{q}_{a b}$. Thus, we see the source of the divergence in (4.5) : the fact that $\vec{N}$ is an asymptotic Killing-vector does not imply that $\bar{E}_{i}^{a}$ is also Lie-annihilated but only that it is asymptotically rotated in the tangent space:

$$
\mathcal{L}_{\vec{N}} \bar{E}_{i}^{a}=\bar{\eta}_{b c}^{a} \xi^{b} \bar{E}_{i}^{c} \text { i.e. } \xi_{a}=\frac{1}{2 \operatorname{det}(\bar{q})} \bar{\eta}_{a b c} \bar{E}_{i}^{b} \bar{E}_{i}^{c}
$$

is $O(1 / r)$ odd or $\mathrm{O}(1)$ even for an asymptotic translation or rotation respectively. In order to isolate the divergence in (4.5) we write

$$
\begin{aligned}
& K_{a}^{i} \mathcal{L}_{\vec{N}} E_{i}^{a}=K_{a}^{i} \mathcal{L}_{\vec{N}}\left(E_{i}^{a}-\bar{E}_{i}^{a}\right)+K_{a}^{i} \mathcal{L}_{\vec{N}} \bar{E}_{i}^{a} \\
= & K_{a}^{i} \bar{\eta}^{a}{ }_{b c} \xi^{b} \bar{E}_{i}^{c}+\text { finite } \\
= & K_{a}^{i} \epsilon^{i j k} \bar{E}_{j}^{a} \bar{E}_{k}^{b} \frac{\xi_{b}}{\sqrt{\operatorname{det}(\bar{q})}}+\text { finite } \\
= & \left(\left[\epsilon^{i j k} K_{a}^{j} \bar{E}_{k}^{a}\right] \bar{E}_{i}^{b}+K_{a}^{i} \epsilon^{i j k}\left[\bar{E}_{j}^{a}-E_{j}^{a}\right] \bar{E}_{k}^{b}\right) \frac{\xi_{b}}{\sqrt{\operatorname{det}(\bar{q})}}+\text { finite } \\
= & \frac{1}{i} \mathcal{G}_{i} \bar{E}_{i}^{b} \frac{\xi_{b}}{\sqrt{\operatorname{det}(\bar{q})}}+\text { finite }
\end{aligned}
$$

where we absorbed terms that are either $O\left(1 / r^{4}\right)$ even or $O\left(1 / r^{3}\right)$ odd into the expression 'finite'. Thus we managed to peel out the contribution that causes trouble in (4.5) : as expected, it is something proportional to the Gauss-constraint and hence plays no role on the constraint-surface.

It is then motivated to consider as a candidate for a well-defined symmetry-generator corresponding to the vector constraint (we use the same label)

$$
H_{a}\left[N^{a}\right]:=2 i \int_{\Sigma} d^{3} x\left[\left(A_{a}^{i}-\Gamma_{a}^{i}\right) \mathcal{L}_{\vec{N}} E_{i}^{a}-\frac{1}{\sqrt{\operatorname{det}(q)}} E_{i}^{a} \xi_{a} \mathcal{G}_{i}\right]
$$

where $\xi_{a}:=1 /(2 \operatorname{det}(q)) \eta_{a b c} E_{i}^{b} \mathcal{L}_{\vec{N}} E_{i}^{c}$. The reader might worry about the highly non-polynomial appearence of (4.9) but, as we will show, the constraints remain polynomial and this is the important thing to keep when thinking of quantizing general relativity ([1]).

Note that we have 'unbarred' everything in (4.9) as compared to (4.8) which we justify now by direct computation, thereby showing that (4.9) is well-defined and differentiable. By simply using the definitions we verify that the integrand of (4.9) is given by

$$
\left(E^{-1}\right)_{b}^{i}\left(A_{a}^{i}-\Gamma_{a}^{i}\right) \mathcal{L}_{\vec{N}}\left(\operatorname{det}(q) q^{a b}\right)
$$

which is $O\left(1 / r^{3}\right)$ odd or $O\left(1 / r^{4}\right)$ even repectively and thus yields a convergent integral thanks to the fact that $\vec{N}$ is an asymptotic Killing vector (recall the discussion 
at the end of the previous section). Thus, we have already established finiteness.

In order to show that (4.9) is also already functionally differentiable, we first display the relation of (4.9) with the constraints. We have

$$
\begin{aligned}
-K_{a}^{i} \mathcal{L}_{\vec{N}} E_{i}^{a}= & -\mathcal{L}_{\vec{N}}\left(K_{a}^{i} E_{i}^{a}\right)+E_{i}^{a} \mathcal{L}_{\vec{N}} K_{a}^{i} \\
= & \left(N^{b} \bar{D}_{b} K_{a}^{i}+K_{b}^{i} \bar{D}_{a} N^{b}\right) E_{i}^{a}-\bar{D}_{b}\left(N^{b} K_{a}^{i} E_{i}^{a}\right) \\
= & N^{a}\left(E_{i}^{b} \bar{D}_{a} K_{b}^{i}-\bar{D}_{b}\left(K_{a}^{i} E_{i}^{b}\right)\right)-2 \bar{D}_{[b}\left(N^{b} K_{a]}^{i} E_{i}^{a}\right) \\
= & -i N^{a}\left(2 E_{i}^{b} \bar{D}_{[a} A_{b]}^{i}-A_{a}^{i} \bar{D}_{b} E_{i}^{b}\right)-i N^{a}\left(2 E_{i}^{b} \bar{D}_{[a} \Gamma_{b]}^{i}-\Gamma_{a}^{i} \bar{D}_{b} E_{i}^{b}\right) \\
& +2 i \bar{D}_{[b}\left(N^{b}\left[A_{a]}^{i}-\Gamma_{a]}\right] E_{i}^{a}\right) .
\end{aligned}
$$

Due to the definition of the spin-connection, we have $0=D_{a} E_{i}^{a}=\bar{D}_{a} E_{i}^{a}+\epsilon_{i j k} \Gamma_{a}^{j} E_{k}^{a}$ so that the bracket of the 2 nd term in the last equation can be written

$$
2 E_{i}^{b} \bar{D}_{[a} \Gamma_{b]}^{i}-\Gamma_{a}^{i} \bar{D}_{b} E_{i}^{b}=E_{i}^{b}\left(2 \bar{D}_{[a} \Gamma_{b]}^{i}+\epsilon_{i j k} \Gamma_{a}^{j} \Gamma_{b}^{k}\right)=E_{i}^{b} R_{a b}^{i} \equiv 0
$$

due to the first Bianchi-identity. Accordingly, (4.11) can be rewritten in the form

$$
-i N^{a}\left(F_{a b}^{i} E_{i}^{b}-A_{a}^{i} \mathcal{G}_{i}\right)+2 i \bar{D}_{[b}\left(N^{b}\left[A_{a]}^{i}-\Gamma_{a]}\right] E_{i}^{a}\right)
$$

where $1 / 2 F$ is the curvature of the Ashtekar-connection and we have used the definition of the Gauss-constraint (4.1). Since the variation of the action with respect to $\Lambda^{i}$ yields the Gauss-constraint, the variation with respect to the shift-vector field results in the diffeomorphism-contraint, i.e. the 1st bracket in (4.13), for a shift corresponding to the generator of a gauge transformation (i.e. the shift is at most a supertranslation at spi). Hence the polynomial form of the constraints is not spoiled by the nonpolynomial term in the full vector contraint

$\left.H_{a}\left[N^{a}\right]=-2 i \int_{\Sigma} d^{3} x\left[N^{a}\left(F_{a b}^{i} E_{i}^{b}-A_{a}^{i} \mathcal{G}_{i}\right)+\frac{1}{\sqrt{\operatorname{det}(q)}} E_{i}^{a} \xi_{a} \mathcal{G}_{i}\right]-4 i \int_{\partial \Sigma} d S_{a}\left[A_{b}^{i}-\Gamma_{b}^{i}\right] N^{[b} E_{i}^{a]}\right)$.

Note that the surface term is not Ashtekar's expression ([1]) which is just the one given in (4.14) but without the second term including the spin-connection. It has the advantage of giving (modulo reality conditions) manifestly the ADM-momentum. For a pure translation the surface term in (4.14) reduces to Ashtekar's expression but, as we will show, this is not true for our boundary conditions when including the asymptotic Lorentz group.

Let us now check functional differentiability of (4.14). Looking at the surface term that is picked up when varying (4.14) we obtain

$$
\begin{aligned}
& \delta H_{a}\left[N^{a}\right]_{\mid \partial \Sigma} \\
= & \int_{\partial \Sigma}\left[-4 i d S_{[a} \delta A_{b]}^{i} N^{a} E_{i}^{b}+2 i d S_{b} A_{a}^{i} \delta E_{i}^{b} N^{a}\right. \\
& -2 i(\operatorname{det}(q))^{-1 / 2}\left(E_{i}^{a} \xi^{a}\right) \delta E_{i}^{b} d S_{b}-i(\operatorname{det}(q))^{-3 / 2} \mathcal{G}_{i} E_{i}^{a} \\
& \eta_{a b c} E_{j}^{b} \mathcal{L}_{\vec{N}} \delta E_{j}^{c} 4 i d S_{[a}\left(\delta A_{b]}^{i}-\delta \Gamma_{b]}^{i}\right) E_{i}^{b} \\
& \left.-4 i d S_{a}\left(A_{b}^{i}-\Gamma_{b}^{i}\right) N^{[b} \delta E^{a]}\right] .
\end{aligned}
$$

The first line comes from the variation of the polynomial part of the volume term, the second from its nonpolynomial part and the last line arises from varying the 
surface term. We observe that the first term of the 1st line cancels against the first term in the first bracket of the last line. Furthermore, the 2nd term of the first line as well as the complete last bracket of the last line and the term proportional to the Gauss-constraint in the middle line vanish identically irrespective of the nature of the tranformation due to parity or fall-off. Recalling the definition of $\xi^{a}$ we thus end up with

$$
\delta H_{a}\left[N^{a}\right]=-2 i \int_{\partial \Sigma} d S_{a}\left[\frac{1}{2[\operatorname{det}(q)]^{3 / 2}} E_{i}^{b} \eta_{b c d} E_{j}^{c} \mathcal{L}_{\vec{N}} E_{j}^{d}-2 N^{[b} \delta\left(E_{i}^{a]} \Gamma_{b}^{i}\right)\right] .
$$

By using the explicit expression (3.10) for the spin-connection in terms of the triads, it is easy to show that

$$
\begin{aligned}
E_{i}^{a} \Gamma_{a}^{i} & =\frac{1}{2} \epsilon^{a b c} e_{c}^{i} \bar{D}_{b} e_{a}^{i} \\
E_{i}^{a} \Gamma_{b}^{i} & =\frac{1}{2} \epsilon^{a c d}\left(\bar{D}_{c} q_{b d}+e_{c}^{i} \bar{D}_{b} e_{d}^{i}\right)
\end{aligned}
$$

Since the terms in which the underivated parts are varied vanish due to parity or fall-off, we arrive after simple algebra at

$$
\begin{aligned}
\delta H_{a}\left[N^{a}\right]= & i \int_{\partial \Sigma}(\operatorname{det}(q))^{1 / 2} d S_{a}\left[\eta ^ { a c d } \left(\frac{1}{2} D_{c} N_{d} q^{e f} \delta q_{e f}\right.\right. \\
& \left.-N^{b}\left(D_{d} \delta q_{b c}-e_{c}^{i} D_{b} \delta e_{d}\right)\right)+\eta^{b c d}\left(e_{b}^{i} \delta e_{i}^{a} D_{c} N_{d}-N^{a} e_{d}^{i} D_{c} \delta e_{b}^{i}\right]
\end{aligned}
$$

where we have used $E_{i}^{a}=\sqrt{q}^{1 / 2} e_{i}^{a}$ in order to write everything in terms of variation of the triads and we could replace $\bar{D} \delta f$ by $D \delta f$ for any f because the additional contributions included in D fall-off one order faster while (4.18) is anyway either $O(1)$ even or $O(r)$ odd.

We will now discuss the 3 possibilities for the fall-off of the shift :

Case 1) supertranslation :

(4.18) is $O(1)$ and odd, so vanishes identically.

Case 2) translation :

We have now $D_{a} N_{b}=O\left(1 / r^{2}\right)$ so there are no contributions from these terms in (4.18), hence

$$
\begin{aligned}
\delta H_{a}\left[N^{a}\right] & =-i \int_{\partial \Sigma}(\operatorname{det}(q))^{1 / 2} d S_{a}\left[\eta^{a c d} e f N^{b}\left(D_{d} \delta q_{b c}-e_{c}^{i} D_{b} \delta e_{d}\right)+\eta^{b c d} N^{a} e_{d}^{i} D_{c} \delta e_{b}^{i}\right] \\
& =i \int_{\partial \Sigma}(\operatorname{det}(q))^{1 / 2} d S_{a}\left[\left(\eta^{b c d} N^{a}-\eta^{a c d} N^{b}\right) e_{d}^{i} D_{b} \delta e_{c}^{i}-\eta^{a c d} N^{b} D_{d} \delta q_{b c}\right]
\end{aligned}
$$

The last term can be written

$$
(\operatorname{det}(q))^{1 / 2} d S_{a} \eta^{a c d} N^{b} D \delta q_{b c}=d x^{c} \wedge d x^{d} D_{d}\left(N^{b} \delta q_{b c}\right)+O(1 / r)=d \wedge\left(N^{b} \delta q_{b c} d x^{c}\right)+O(1 / r)
$$

and thus drops out since $\partial \partial \Sigma=\emptyset$. The first term on the other hand can be cast into the form

$$
\begin{aligned}
& (\operatorname{det}(q))^{1 / 2} d S_{a}\left(\eta^{b c d} N^{a}-\eta^{a c d} N^{b}\right) e_{d}^{i} D_{b} \delta e_{c}^{i} \\
= & (\operatorname{det}(q))^{1 / 2} d S_{a} D_{b}\left[\left(\eta^{b c d} N^{a}-\eta^{a c d} N^{b}\right) e_{d}^{i}\right] \delta e_{c}^{i} \\
& +O(1 / r)=(\operatorname{det}(q))^{1 / 2} d S_{a} D_{b} \eta^{a b c} f_{c}+O(1 / r)=d \wedge f+O(1 / r)
\end{aligned}
$$


where we have defined the 1-form $f_{e}:=-1 / 2 \eta_{e a b}\left(\eta^{b c d} N^{a}-\eta^{a c d} N^{b}\right) e_{d}^{i} \delta e_{c}^{i}$. Hence, (4.19) is just the integral of an exact 1-form over an exact chain and thus vanishes identically.

case 3) rotation :

We now exploit $D_{a} N_{b}=\eta_{a b c} \xi^{c}, D_{a} \xi^{c}=O\left(1 / r^{2}\right)$. The idea is to integrate by parts in (4.19) and to order terms proportional to $N^{a}$ or $D_{a} N^{b}$. We thus have, using $D_{a} e_{b}^{i}=0$,

$$
\begin{aligned}
\delta H_{a}\left[N^{a}\right]= & i \int_{\partial \Sigma}(\operatorname{det}(q))^{1 / 2} d S_{a}\left[\eta ^ { a c d } \left(\frac{1}{2} q^{e f} \delta q_{e f} D_{c} N_{d}+\delta q_{b c} D_{d} N^{b}\right.\right. \\
& \left.-e_{c}^{i} \delta e_{d} D_{b} N^{b}\right)+\eta^{b c d}\left(e_{b}^{i} \delta e_{i}^{a} D_{c} N_{d}+e_{d}^{i} \delta e_{b}^{i} D_{c} N^{a}\right) \\
& +\eta^{a c d}\left(-D_{d}\left(\delta q_{b c} N^{b}\right)+D_{b}\left(e_{c}^{i} \delta e_{d} N^{b}\right)-\eta^{b c d} D_{c}\left(e_{d}^{i} \delta e_{b}^{i} D_{c} N^{a}\right)\right]
\end{aligned}
$$

We can write the last two brackets as folows:

$$
\begin{aligned}
& (\operatorname{det}(q))^{1 / 2} d S_{a}\left[-\eta^{a c d} D_{d}\left(\delta q_{b c} N^{b}\right)-D_{b}\left(\left[\eta^{a c d} N^{b}-\eta^{b c d} N^{a}\right] e_{d}^{i} \delta e_{c}\right)\right] \\
= & (\operatorname{det}(q))^{1 / 2} d S_{a}\left[-\eta^{a c d} D_{d}\left(\delta q_{b c} N^{b}\right)-\eta^{a b e} D_{b}\left(\eta_{e f g} \eta^{f c d} N^{g} e_{d}^{i} \delta e_{c}\right)\right] \\
= & (\operatorname{det}(q))^{1 / 2} d S_{a} \eta^{a b e} D_{b}\left[\delta q_{e c} N^{c}-\eta_{e f g} \eta^{f c d} N^{g} e_{d}^{i} \delta e_{c}\right]
\end{aligned}
$$

and thus displays it as an exact 2-form. Since we have regularized the surface integrals in such a way (see the remark in section 2) that they are to be evaluated at finite $r$ and then one takes the limit, we see that due to the fact that the sphere at finite $\mathrm{r}$ is also without boundary, the integral over (4.23) drops out of (4.22). We are thus left with the first two brackets in (4.22). These turn out to cancel each other algebraically when using the above expression for $D_{a} N_{b}$ :

$$
\begin{aligned}
& \eta^{a c d}\left(\frac{1}{2} q^{e f} \delta q_{e f} D_{c} N_{d}+\delta q_{b c} D_{d} N^{b}-e_{c}^{i} \delta e_{d} D_{b} N^{b}\right)+\eta^{b c d}\left(e_{b}^{i} \delta e_{i}^{a} D_{c} N_{d}+e_{d}^{i} \delta e_{b}^{i} D_{c} N^{a}\right) \\
= & \xi^{a} q^{e f} \delta q_{e f}+2 q_{e}^{[a} q_{f}^{c]} \xi^{f} q^{e b} \delta q_{b c}-2 \xi^{b} e_{i}^{a} \delta e_{b}^{i}+2 q_{e}^{[d} q_{f}^{b]} \xi^{f} q^{a e} e_{d}^{i} \delta e_{b}^{i} \\
= & q^{a b} \xi^{c} \delta q_{b c}-\xi^{b} e_{i}^{a} \delta e_{b}^{i}-\xi^{d} q^{a b} e_{d}^{i} \delta e_{b}^{i} \\
= & q^{a b} \xi^{c}\left(\delta q_{b c}-e_{c}^{i} \delta e_{b}^{i}\right)-\xi^{b} e_{i}^{a} \delta e_{b}^{i} \\
= & q^{a b} \xi^{c} e_{b}^{i} \delta e_{c}^{i}-\xi^{b} e_{i}^{a} \delta e_{b}^{i} \equiv 0
\end{aligned}
$$

where in the last step we used $\delta q_{a b}=2 e_{(a}^{i} \delta e_{b)}^{i}$.

Thus we proved that (4.14) is finite, functionally differentiable, reduces to the ADMmomentum on the constraint surface and its (weakly) vanishing part is polynomial in the basic variables. It therefore satisfies all requirements that one wishes to impose on a symmetry generator corresponding to momentum. We will prove in the next section that (4.14) generates the correct symmetries and gauge transformations.

Note :

In principle one could have found the correct version (4.14) of the vector-constraint in terms of Ashtekar's variables also simply by carefully implementing the Gaussconstraint thus displaying (4.14) exactly as the old ADM-momentum expression after doing the complex canonical transformation which leads to Ashtekar's theory : starting again from $(2.8)$ we have

$$
\int_{\Sigma} d^{3} x p^{a b} \mathcal{L}_{\vec{N}} q_{a b}=-\int_{\Sigma} d^{3} x p_{a b} \mathcal{L}_{\vec{N}} q^{a b}
$$




$$
\begin{aligned}
& =-\int_{\Sigma} d^{3} x \sqrt{\operatorname{det}(q)}\left(K_{a b}-K q_{a b}\right) \mathcal{L}_{\vec{N}} q^{a b} \\
& =-\int_{\Sigma} d^{3} x \frac{1}{\sqrt{\operatorname{det}(q)}}\left(K_{a b} \operatorname{det}(q) \mathcal{L}_{\vec{N}} q^{a b}-K \mathcal{L}_{\vec{N}} \operatorname{det}(q)\right) \\
& =-\int_{\Sigma} d^{3} x \frac{1}{\sqrt{\operatorname{det}(q)}} K_{(a b)} \mathcal{L}_{\vec{N}}\left(\operatorname{det}(q) q^{a b}\right)
\end{aligned}
$$

and it is important to see that only the symmetric part of the tensor $K_{a b}$ contributes to (4.25). The asymmetric part on the other hand is directly related to the Gauss constraint : using the definition of $A_{a}^{i}, K_{a}^{i}:=K_{a b} e_{i}^{b}$ and $D_{a} E_{i}^{a}=0$ we have

$$
\mathcal{G}_{i}=i \sqrt{\operatorname{det}(q)} \epsilon_{i j k} e_{j}^{b} e_{k}^{a} K_{a b}=-i \epsilon^{a b c} K_{a b} e_{c}^{i}
$$

and thus we conclude

$$
\begin{aligned}
\int_{\Sigma} d^{3} x p^{a b} \mathcal{L}_{\vec{N}} q_{a b} & =-2 \int_{\Sigma} d^{3} x \frac{1}{\sqrt{\operatorname{det}(q)}} K_{(a b)} \mathcal{L}_{\vec{N}}\left(E_{i}^{a}\right) E_{i}^{b} \\
& =-2 \int_{\Sigma} d^{3} x\left[K_{a b}-K_{[a b]}\right] \mathcal{L}_{\vec{N}}\left(E_{i}^{a}\right) e_{i}^{b} \\
& =-2 \int_{\Sigma} d^{3} x\left[K_{a}^{i}-\frac{1}{2} \epsilon_{a b c} \epsilon^{d e c} K_{d e} e_{i}^{b}\right] \mathcal{L}_{\vec{N}}\left(E_{i}^{a}\right) \\
& =-2 \int_{\Sigma} d^{3} x\left[K_{a}^{i}-\frac{i}{2} \epsilon^{i j k} E_{a}^{k} \mathcal{G}_{j}\right] \mathcal{L}_{\vec{N}}\left(E_{i}^{a}\right) \\
& =2 i \int_{\Sigma} d^{3} x\left[K_{a}^{i} \mathcal{L}_{\vec{N}}\left(E_{i}^{a}\right)+\frac{1}{2}\left(\epsilon^{i j k} \mathcal{L}_{\vec{N}}\left(E_{j}^{a}\right) E_{a}^{k}\right) \mathcal{G}_{i}\right]
\end{aligned}
$$

which is precisely (4.14).

We finally come to discuss energy. Again we follow the strategy to take the old ADM expression (2.11), write it in terms of Ashtekar's variables and look for the necessary modifications. Writing the Ashtekar-connection in terms of the spin-connection and the extrinsic curvature, it is easy to show that (compare [1]) the ADM- scalar constraint turns out to be

$$
\begin{aligned}
C[\underset{\sim}{N}] & \left.=\int_{\Sigma} d^{3} x \underset{\sim}{\sim}\left[F_{a b}^{i} \epsilon_{i j k} E_{j}^{a} E_{k}^{b}+2 D_{a}\left(E_{i}^{a} \mathcal{G}_{i}\right)\right)\right] \\
& \left.=\int_{\Sigma} d^{3} x \underset{\sim}{\sim}\left[F_{a b}^{i} \epsilon_{i j k} E_{j}^{a} E_{k}^{b}+2 \bar{D}_{a}\left(E_{i}^{a} \mathcal{G}_{i}\right)\right)\right]
\end{aligned}
$$

(we used the fact that the divergence of a vector density is independent of the metric connection) and in [2] the necessary counterterms are obtained simply by integrating by parts this expression an appropriate number of times in order to arrive at a manifestly finite expression. We follow this approach here.

We observe that the part proportional to $\bar{D} A$ in the first term and the 2 nd term in (4.28) are either $O\left(1 / r^{3}\right)$ even or $O\left(1 / r^{2}\right)$ odd so they diverge while the remainder is already finite. We are now going to integrate by parts both divergent terms :

$$
C[\underset{\sim}{\sim}]=\int_{\Sigma} d^{3} x\left[-2 A_{b}^{i} \bar{D}_{a}\left(\underset{\sim}{\sim} \epsilon_{i j k} E_{j}^{a} E_{k}^{b}\right)-2 E_{i}^{a} \mathcal{G}_{i} D_{a} \underset{\sim}{\sim} \underset{\sim}{\sim} A_{a}^{j} A_{b}^{k} \epsilon_{i j k} E_{m}^{a} E_{n}^{b} \epsilon^{i m n}\right]
$$




$$
\begin{aligned}
& +\int_{\partial \Sigma} d S_{a} 2 \underset{\sim}{\sim}\left[A_{b}^{i} \epsilon_{i j k} E_{j}^{a} E_{k}^{b}+E_{i}^{a} \mathcal{G}^{i}\right] \\
= & \int_{\Sigma} d^{3} x\left[-2\left(D_{a} \underset{\sim}{N} E_{i}^{a} \bar{D}_{b} E_{i}^{b}+\underset{\sim}{\sim}\left(-2 A_{b}^{i} \epsilon_{i j k} \bar{D}_{a}\left(E_{j}^{a} E_{k}^{b}\right)+A_{a}^{j} A_{b}^{k} \epsilon_{i j k} E_{m}^{a} E_{n}^{b} \epsilon^{i m n}\right)\right]\right. \\
& +\int_{\partial \Sigma} d S_{a} 2 \underset{\sim}{N} E_{i}^{a} \bar{D}_{b} E^{b}
\end{aligned}
$$

where we have used the definition of the Gauss-constraint (4.1). While the term proportional to $\underset{\sim}{N}$ in the volume integral is already manifestly convergent, the term proportional $D_{a} \underset{\sim}{\sim}$ is not. However, we write in the same term $\bar{D}_{b} E_{i}^{b}=\bar{D}_{b}\left(E_{i}^{b}-\bar{E}_{i}^{b}\right)$ and integrate by parts again. Note that $\bar{D}_{b} D_{a} N=(\operatorname{det}(q))^{-1 / 2} \bar{D}_{a} \bar{D}_{b} N+O\left(1 / r^{2}\right)$ is of order $O\left(1 / r^{2}\right)$ at least, even for a boost $\left(\partial\left(x_{\text {cart }}\right)^{b} / \partial \bar{x}^{a}\right.$ is of order one and in the cartesian frame $\left(x_{\text {cart }}\right)^{a}$ the assertion is obvious). We thus obtain

$$
\begin{aligned}
C[\underset{\sim}{N}]= & \int_{\Sigma} d^{3} x\left[-2 \bar{D}_{b}\left(\left(D_{a} \underset{\sim}{N}\right) E_{i}^{a}\right)\left(E_{i}^{b}-\bar{E}_{i}^{b}\right)+\underset{\sim}{N}\left(-2 A_{b}^{i} \epsilon_{i j k} \bar{D}_{a}\left(E_{j}^{a} E_{k}^{b}\right)\right.\right. \\
& \left.\left.+A_{a}^{j} A_{b}^{k} \epsilon_{i j k} E_{m}^{a} E_{n}^{b} \epsilon^{i m n}\right)\right]+\int_{\partial \Sigma} d S_{a} 2\left[N E_{i}^{a} \bar{D}_{b} E^{b}\right. \\
& \left.-\left(D_{b} N\right) E_{i}^{b}\left(E_{i}^{a}-\bar{E}_{i}^{a}\right)\right]
\end{aligned}
$$

and now the volume integral is manifestly convergent and thus gives rise to the ansatz for the correct symmetry generator :

$$
H[\underset{\sim}{\sim}]:=C[\underset{\sim}{N}]-\int_{\partial \Sigma} d S_{a} 2\left[\underset{\sim}{\sim} E_{i}^{a} \bar{D}_{b} E^{b}-\left(D_{b} \underset{\sim}{\sim}\right) E_{i}^{b}\left(E_{i}^{a}-\bar{E}_{i}^{a}\right)\right] .
$$

In fact, it is easy to prove that (4.31) is already differerentiable. The boundary contribution of the volume term is given by

$$
\begin{aligned}
\delta C[N]_{\mid \text {boundary term }}= & 2 \int_{\partial \Sigma} d S_{a} \underset{\sim}{\sim}\left[\epsilon_{i j k} E_{j}^{a} E_{k}^{b} \delta A_{b}^{i}+\delta\left(E_{i}^{a} \mathcal{G}_{i}\right)\right] \\
& -\left\{2 \int_{\Sigma} d^{3} x \bar{D}_{a} N \delta\left(E_{i}^{a} \mathcal{G}_{i}\right)\right\}_{\mid b o u n d a r y} \text { term } \\
= & 2 \int_{\partial \Sigma} d S_{a} \underset{\sim}{N}\left[\epsilon_{i j k} E_{j}^{a} E_{k}^{b} \delta A_{b}^{i}+E_{i}^{a}\left(\bar{D}_{b} \delta E_{i}^{b}\right.\right. \\
& \left.+\epsilon_{i j k} E_{k}^{b} \delta A_{b}^{j}\right]-\left\{2 \int_{\Sigma} d^{3} x \bar{D}_{a} N E_{i}^{a} \bar{D}_{b} \delta\left(E_{i}^{b}\right\}_{\mid b o u n d a r y}\right. \text { term } \\
= & 2 \int_{\partial \Sigma} d S_{a}\left[\underset{\sim}{N} E_{i}^{a} \bar{D}_{b} \delta E_{i}^{b}-D_{b} \underset{\sim}{\sim} E_{i}^{b} \delta E_{i}^{a}\right]
\end{aligned}
$$

where we could neglect all terms that are of order $O\left(1 / r^{3}\right)$ (in particular we could replace $\bar{D}$ by D). Hence we have for the variation of the surface integral in (4.31)

$$
-\delta C[\underset{\sim}{N}]_{\mid \text {boundary term }}-2 \int_{\partial \Sigma} d S_{a}\left[\underset{\sim}{\sim} \delta E_{i}^{a} \bar{D}_{b} E^{b}-\left(D_{b} \underset{\sim}{\sim}\right) \delta E_{i}^{b}\left(E_{i}^{a}-\bar{E}_{i}^{a}\right)\right]
$$

and the integrand of the latter integral is easily seen to be of order $O\left(1 / r^{2}\right)$ even or $O\left(1 / r^{3}\right)$ odd and thus vanishes identically.

This completes the proof that expression (4.31) is differentiable.

Let us check that the boundary term in (4.31) is indeed the expression (2.11) given 
in section 2. We have

$$
\begin{aligned}
E_{i}^{a} \bar{D}_{b} E_{i}^{b} & =\sqrt{\operatorname{det}(q)} e_{i}^{a} \bar{D}_{b}\left(\sqrt{\operatorname{det}(q)} e_{i}^{b}\right) \\
& =\operatorname{det}(q)\left(E_{i}^{a} \bar{D}_{b} e_{i}^{b}+\frac{1}{2} q^{a b} q^{c d} \bar{D}_{b} q_{c d}\right) \\
& =\operatorname{det}(q) q^{a c}\left(e_{c}^{i} \bar{D}_{b} e_{i}^{b}+\frac{1}{2} q^{b d} \bar{D}_{c} q_{b d}\right) \\
& =\operatorname{det}(q) q^{a c} q^{b d}\left(-e_{d}^{i} \bar{D}_{b} e_{c}^{i}+\frac{1}{2} \bar{D}_{c} q_{b d}\right)
\end{aligned}
$$

whence (remember that $\underset{\sim}{\sim} \sqrt{\operatorname{det}(q)}=N$ is the lapse), by using the the Gaussconstraint,

$$
\begin{aligned}
& -\int_{\partial \Sigma} d S_{a} 2 \underset{\sim}{\sim} A_{b}^{i} \epsilon_{i j k} E_{j}^{a} E_{k}^{b} \\
\approx & -\int_{\partial \Sigma} d S_{a} 2 \underset{\sim}{\sim} E_{i}^{a} \bar{D}_{b} E^{b} \\
= & \int_{\partial \Sigma} d S_{d} N \sqrt{\operatorname{det}(q)} q^{a c} q^{b d}\left(2 e_{a}^{i} \bar{D}_{c} e_{b}^{i}-\bar{D}_{c} q_{b d}\right) \\
= & \int_{\partial \Sigma} d S_{d} N \sqrt{\operatorname{det}(q)} q^{a c} q^{b d}\left(\left[\bar{D}_{c} q_{a b}-\bar{D}_{c} q_{b d}\right]+2 e_{[a}^{i} \bar{D}_{|c|} e_{b]}^{i}\right) \\
= & E+\int_{\partial \Sigma} d S_{d} \sqrt{\operatorname{det}(q)} q^{a c} q^{b d} 2 e_{[a}^{i} \bar{D}_{|c|} e_{b]}^{i}
\end{aligned}
$$

and the integrand of the second term in (4.36) can be shown to vanish to 2 nd order in $1 / r$ by virtue of the symmetry of $f_{a b}$ (recall $\left.(2.1)\right)$ :

$$
\begin{aligned}
e_{[a}^{i} \bar{D}_{|c|} e_{b]}^{i} & =\left(\bar{e}_{[a}^{i}+\frac{f_{[a}^{i}}{r}+O(1 / r)\right)\left(\bar{D}_{|c|} \frac{f_{b]}^{i}}{r}+O\left(1 / r^{3}\right)\right) \\
& =\bar{e}_{[a}^{i}\left(\bar{e}_{i}^{d} \bar{D}_{|c|} \frac{f_{b] d}}{2 r}+\frac{f_{b] d}}{2 r} \bar{D}_{c} \bar{e}_{i}^{d}\right)+O\left(1 / r^{3}\right) \\
& =\bar{q}_{[a}^{d} \bar{D}_{|c|} \frac{f_{b] d}}{2 r}+O\left(1 / r^{3}\right) \\
& =\bar{D}_{c} \frac{f_{[b a]}}{2 r}+O\left(1 / r^{3}\right)=O\left(1 / r^{3}\right)
\end{aligned}
$$

since $\bar{D} \bar{q}_{a b}=\bar{D} \bar{e}_{i}^{a}=0$. Hence we have already reproduced the ADM-energy.

As far as the second contribution in (4.31) is concerned we first note that the $O(1)$ contribution is odd and thus integrates to zero. Hence we may replace any quantity of the integrand by another term which differs from the original one by a term which is higher by one order of $1 / r$. We thus write first with a glimpse at $(2.11)$

$$
\begin{array}{r}
\int_{\partial \Sigma} d S_{a} 2\left(D_{b} \underset{\sim}{\sim}\right) E_{i}^{b}\left(E_{i}^{a}-\bar{E}_{i}^{a}\right)=\int_{\partial \Sigma} d S_{a} 2\left(D_{b} N\right) \\
e_{i}^{b}\left(e_{i}^{a}-\sqrt{\frac{\operatorname{det}(\bar{q})}{\operatorname{det}(q)}} \bar{e}_{i}^{a}\right)=\int_{\partial \Sigma} d S_{a} 2\left(D_{b} N\right)\left(q^{a b}-\sqrt{\frac{\operatorname{det}(\bar{q})}{\operatorname{det}(q)}} e_{i}^{b} \bar{e}_{i}^{a}\right)
\end{array}
$$

and we expand the second term in (4.38) according to (3.1) :

$$
\left.\left.\sqrt{\frac{\operatorname{det}(\bar{q})}{\operatorname{det}(q)}} e_{i}^{b} \bar{e}_{i}^{a}\right)=\sqrt{\frac{1}{\operatorname{det}\left(\bar{q}^{-1} q\right)}}\left(\bar{e}_{i}^{b}-\bar{q}^{b c} \frac{f_{c d}}{2 r} \bar{e}_{i}^{d}\right) \bar{e}_{i}^{a}\right)+O\left(1 / r^{2}\right)
$$




$$
\begin{aligned}
& =\left(1-\frac{\bar{q}^{e f} f_{e f}}{2 r}\right)\left(\bar{q}^{a b}-\bar{q}^{b c} \frac{f_{c d}}{2 r} \bar{q}^{a d}\right)+O\left(1 / r^{2}\right) \\
& =\bar{q}^{a b}-\frac{1}{2 r}\left(q^{a c} q^{b d}+q^{a b} q^{c d}\right) f_{c d}+O\left(1 / r^{2}\right) .
\end{aligned}
$$

Now, using that $f_{c d}=r\left(q_{c d}-\bar{q}_{c d}\right)+O(1 / r)$ we find that (4.38) can indeed be written

$$
\begin{aligned}
& \int_{\partial \Sigma} d S_{a}\left(D_{b} \underset{\sim}{\sim}\right)\left(q^{a c} q^{b d}-q^{a b} q^{c d}\right) \frac{f_{c d}}{r} \\
& \int_{\partial \Sigma} d S_{d}\left(D_{c} \underset{\sim}{\sim}\right)\left(q^{d b} q^{c a}-q^{d c} q^{b a}\right) \frac{f_{b a}}{r} \\
& \int_{\partial \Sigma} d S_{d}\left(D_{c} N\right) q^{a b} q^{c d}\left(q_{a[b}-\bar{q}_{a[b}\right) D_{c]} N
\end{aligned}
$$

and we recover exactly $(2.11)$.

Hence we succeeded in giving a differentiable and finite expression which reproduces the surface terms of the old ADM-theory.

\section{The symmetry-algebra}

We finally come to compute the Poisson-structure of the generators (4.1), (4.14) and (4.31). The Poisson-structure for Lagrange-multipliers $\Lambda^{i}, N^{a}$ and $\underset{\sim}{N}$ corresponding to pure gauge transformations was already given in [5].

As in [2] it turns out that even for Lagrange multiplicators corresponding to symmetries the symmetry algebra just equals the gauge algebra.

Since the transition from the ADM-variables to the new variables is a canonical transformation, we can copy from [2] the algebra restricted to the vector- and scalar constraint because in section 4 we showed that our expressions reduce exactly to the ADM expressions according to this canonical transformation. The only new brackets are those including a Gauss-constraint. Let us display the complete variation of the symmetry generators :

$$
\begin{aligned}
\delta \mathcal{G}_{i}\left[\Lambda^{i}\right]= & \int_{\Sigma} d^{3} x\left[\left(-\bar{D}_{a} \Lambda^{i}+\epsilon_{i j k} \Lambda^{j} A_{a}^{k}\right) \delta E_{i}^{a}-\epsilon_{i j k} \Lambda^{j} E_{k}^{a}\right) \delta A_{a}^{i} \\
\delta H_{a}\left[N^{a}\right]= & \int_{\Sigma} d^{3} x\left[\left(\mathcal{L}_{\vec{N}} A_{a}^{i}+\mathcal{L}_{\vec{N}}\left(\epsilon_{i j k} E_{a}^{j} \mathcal{G}_{k}\right)-\left(\mathcal{G}_{i}\right) \epsilon^{i j k}\right.\right. \\
& \left.\left(\mathcal{L}_{\vec{N}} E_{j}^{b} E_{a}^{k}\right) E_{b}^{i}-\left(-\bar{D}_{a} \Lambda[\vec{N}]_{i}+\epsilon_{i j k} \Lambda[\vec{N}]^{j} A_{a}^{k}\right)\right) \delta E_{i}^{a} \\
& \left.-\left(\mathcal{L}_{\vec{N}} E_{i}^{a}+\epsilon_{i j k} \Lambda[\vec{N}]_{j} E_{k}^{a}\right) \delta A_{a}^{i}\right] \\
& \delta H[\underset{\sim}{N}]=\int_{\Sigma} d^{3} x\left[2\left(\mathcal{D}_{b}\left(N \epsilon_{i j k} E_{j}^{a} E_{k}^{b}\right)+\epsilon_{i j k} \Lambda[N]_{j} E_{k}^{a}\right) \delta A_{a}^{i}\right. \\
& -2\left(\stackrel{N}{\sim} \epsilon_{i j k} F_{a b}^{j} E_{k}^{b}+\left(\bar{D}_{a} \underset{\sim}{N}\right) \mathcal{G}_{i}+\left(-\bar{D}_{a} \Lambda[\underset{\sim}{N}]_{i}\right.\right. \\
& \left.\left.\left.+\epsilon_{i j k} \Lambda[N]^{j} A_{a}^{k}\right)\right) \delta E_{i}^{a}\right]
\end{aligned}
$$

where we have defined

$$
\begin{aligned}
\Lambda[\vec{N}]^{i} & :=\frac{1}{2} \epsilon^{i j k}\left(\mathcal{L}_{\vec{N}} E_{j}^{a}\right) E_{a}^{k}, \\
\Lambda[\underset{\sim}{N}]^{i}: & =-2\left(D_{a} \underset{\sim}{\sim}\right) E_{i}^{a} .
\end{aligned}
$$


Then it may be checked by explicit calculation that

$$
\begin{aligned}
\left\{\mathcal{G}_{i}\left[\Lambda^{i}\right], \mathcal{G}_{j}\left[\Xi^{j}\right]\right\} & =-i \mathcal{G}_{i}\left[\epsilon_{i j k} \Lambda^{j} \Xi^{k}\right] \\
\left\{\mathcal{G}_{i}\left[\Lambda^{i}\right], H_{a}\left[N^{a}\right]\right\} & =0 \\
\left\{\mathcal{G}_{i}\left[\Lambda^{i}\right], H[N]\right\} & =0 \\
\left\{H_{a}\left[M^{a}\right], H_{b}\left[N^{b}\right]\right\} & =i H_{a}\left[\left(\mathcal{L}_{\vec{M}} \vec{N}\right)^{a}\right] \\
\left\{H_{a}\left[M^{a}\right], H[N]\right\} & =-i H\left[M^{a} D_{a} \stackrel{\sim}{\sim}\right] \\
\{H[M], H[\underset{\sim}{\sim}]\} & =-i H\left[E_{i}^{a} E_{i}^{b}\left(\underset{\sim}{\sim} D_{b} N-\underset{\sim}{\sim} D_{b} M\right)\right]
\end{aligned}
$$

according to the rule that

$$
\left\{A_{a}^{i}(x), E_{b}^{b}(y)\right\}=i \delta_{a}^{b} \delta_{j}^{i} \delta(x, y),\left\{A_{a}^{i}(x), A_{b}^{j}(y)\right\}=\left\{E_{i}^{a}(x), E_{j}^{b}(y)\right\}=0
$$

These equations mean the following (see [2]) : Let

$$
\Phi[N]:=\mathcal{G}_{i}\left[N^{i}\right]+H_{a}\left[N^{a}\right]+H[\underset{\sim}{\sim}]
$$

and compute the Poisson bracket $\{\Phi[M], \Phi[N]\}$ according to (5.4). Then we have the following combinations (the Lagrange-multiplier of the Gauss-constraint is always pure gauge, i.e. $O\left(1 / r^{2}\right)$ even) :

1) $\mathrm{M}$ and $\mathrm{N}$ both pure gauge (odd supertranslations). Then

$\mathcal{L}_{\vec{M}} \vec{N}, M^{a} D_{a} \underset{\sim}{\sim}$ and $E_{i}^{a} E_{i}^{b} \underset{\sim}{\sim} D_{b} \underset{\sim}{\sim}$ are again pure gauge while $M^{i} N^{j}$ is again of even parity when $M^{i}, N^{j}$ are, so the gauge algebra closes, we have a first class system.

2) $\mathrm{M}$ a symmetry, $\mathrm{N}$ pure gauge.

Case a) : M a translation. Then $\mathcal{L}_{\vec{M}} \vec{N}, M^{a} D_{a} \underset{\sim}{N}$ and $E_{i}^{a} E_{i}^{b} \underset{\sim}{\sim} D_{b} \underset{\sim}{\sim}$ (and their counterparts obtained by interchanging $\mathrm{M}$ and $\mathrm{N})$ are $O(1 / r)$ even so the surface integrals vanish identically.

Case b) : $\mathrm{M}$ a Lorentz rotation. Then $\mathcal{L}_{\vec{M}} \vec{N}, M^{a} D_{a} \underset{\sim}{N}$ and $E_{i}^{a} E_{i}^{b} \underset{\sim}{\sim} D_{b} \underset{\sim}{N}$ etc. are $O(1)$ odd and thus the surface integrals also vanish identically.

This means that the Poisson generators have weakly vanishing brackets with the gauge generators, i.e. they are observables in the sense of Dirac.

3) $\mathrm{M}$ and $\mathrm{N}$ both symmetries. Then $\mathcal{L}_{\vec{M}} \vec{N}, M^{a} D_{a} \underset{\sim}{\sim}$ and $E_{i}^{a} E_{i}^{b} \underset{\sim}{\sim} D_{b} \underset{\sim}{\sim}$ etc. display the Poisson algebra.

Accordingly, we managed to reproduce the results of [2] in the new variables.

\section{References}

[1] A. Ashtekar, Phys. Rev. D36 (1987)1587;

A. Ashtekar, New Perspepectives in Canonical Gravity (Monographs and Textbooks in Physical Science, Bibliopolis, Napoli, 1988);

A. Ashtekar, Lectures on Non-Perturbative Canonical Gravity (World Scientific, Singapore, 1991)

[2] R. Beig, N. o Murchadha, Ann. Phys. 174(1987)463

[3] A. Ashtekar, R. O. Hansen, Journ. Math. Phys. 19(1978) 1542 
[4] T. Thiemann, H.A. Kastrup, Nucl. Phys. B399(1993)211

T. Thiemann, PITHA 93-32, August 93, RWTH Aachen, Germany

[5] P.A.M. Dirac, Lectures on Quantum Mechanics (Belfer Graduate School of Science, Yeshiva University, New York, 1964)

[6] Y. Choquet-Bruhat, in: Relativity, Groups and Topology II, ed. by B.S. DeWitt and R. Stora, (North Holland, Amsterdam, 1984)p. 739

[7] R. Beig, B. G. Schmidt, Comm. Math. Phys. 87(1982) 65 